\title{
Probing structural chirality of crystals using high-order harmonic generation in solids
}

\author{
Zi-Yu Chen $\odot^{1, *}$ and Rui Qin ${ }^{2, \dagger}$ \\ ${ }^{1}$ Key Laboratory of High Energy Density Physics and Technology (MoE), College of Physics, Sichuan University, Chengdu 610064, China \\ ${ }^{2}$ National Key Laboratory of Shock Wave and Detonation Physics, Institute of Fluid Physics, China Academy of Engineering Physics, \\ Mianyang 621999, China
}

(Received 10 February 2020; accepted 27 April 2020; published 13 May 2020)

\begin{abstract}
Structural chirality plays an important role in solid-state physics and leads to a variety of novel physics. The feasibility of probing structural chirality of crystals using high harmonic generation in solids is explored in this work. Through first-principles calculations based on the time-dependent density-functional theory framework, we demonstrate that evident circular dichroism (CD) effects can be induced in the high harmonic spectra from a chiral crystal—bulk tellurium. The CD signal reverses for crystals with opposite structural chirality. Besides, the high harmonic spectroscopy also provides an all-optical method for probing lattice symmetry properties and determining orientation of the tellurium crystal.
\end{abstract}

DOI: 10.1103/PhysRevA.101.053423

\section{INTRODUCTION}

Chirality is a property of asymmetry in matter which can exist at every length scale and plays an important role in various branches of science [1]. An object is chiral if it cannot be superposed onto its mirror image, with human hands as one of the examples. The characterization of chirality has been the subject of intensive studies.

Several chiral-sensitive spectroscopy techniques utilizing interaction with circularly polarized light have been developed for discrimination of chiral molecules. For instance, circular dichroism (CD) of photoabsorption spectroscopy in the condensed phase [2] and photoelectron circular dichroism (PECD) in the gas phase $[3,4]$ are widely used for detecting chiral chemical and biological molecules. Besides, nonlinear optical processes in the perturbative regime have been explored to probe chirality in solution and on surfaces [5]. Recently, nonperturbative high harmonic generation (HHG) from chiral gas molecules has also been demonstrated to be chirality sensitive [6-11], thus providing an attractive approach to detect chiral structures and resolve ultrafast chiral dynamics in the gas phase.

Chirality also plays a critical role in solid-state physics. Out of the 230 space groups for nonmagnetic materials, 65 space groups represent structurally chiral crystals [12]. Structurally chirality can lead to a variety of novel physics, such as multiferroicity [13], magnetochiral dichroism [2], skyrmions [14], current-induced magnetizations [15], circular photogalvanic effect [16], and universal topological electronic properties $[17,18]$. Thus it is of great interest to detect and characterize the structural chirality embedded in crystals.

The recent advent of HHG in solids $[19,20]$ has opened up exciting opportunities for not only the development of novel solid-state extreme-ultraviolet and attosecond photonics with

\footnotetext{
*ziyuch@scu.edu.cn

†qinrui.phy@outlook.com
}

bulk [21-25] or at nanoscale [26-36], but also the investigation of strong-field and ultrafast dynamics in the condensed phase using high harmonic spectroscopy [37-40]. Substantial progress has been reported, such as the demonstration of probing atomic-scale crystal structures [41], reconstructing electronic band structures $[42,43]$, measuring Berry curvature [44], characterizing complex ultrafast many-body dynamics in strongly correlated systems [45], and revealing strong-field physics in topological systems [46,47]. Like its counterpart in chiral gas harmonics, chiral HHG in solids may also be useful in revealing chiral structures and dynamics in the condensed phase.

In this work, we extend the chiral high harmonic spectroscopy to solids. The feasibility of probing the structural chirality of crystals using HHG in solids is explored. Through first-principles calculations based on the time-dependent density-functional theory (TDDFT) framework, we demonstrate that considerable CD signals in the HHG spectra can be induced when a crystal with chiral lattice interacts with circularly polarized light. In addition, the high harmonic spectroscopy also provides an optical method to probe lattice symmetry properties and determine orientation of the crystal.

\section{CRYSTAL STRUCTURE}

We choose the simplest material with a chiral structure, i.e., elemental tellurium, to study the structural chirality in crystals. Tellurium has a trigonal crystal structure with three atoms in the unit cell. The unique structure consists of helical chains arranged in a hexagonal array spiraling around axes parallel to the $z$ axis ([0001] direction) at the corners and center of the hexagonal elementary cell, as shown in Fig. 1. Depending on the screw direction of the helical chains, there are two enantiomorphic crystal structures with space groups $P 3_{2} 21$ (no. 154, left-handed) and $P 3_{1} 21$ (no. 152, right-handed) [48]. Each tellurium atom is covalently bonded with its two nearest neighbors on the same chain while forming weak van der Waals-like interchain bonds with its four next-nearest 
(a)

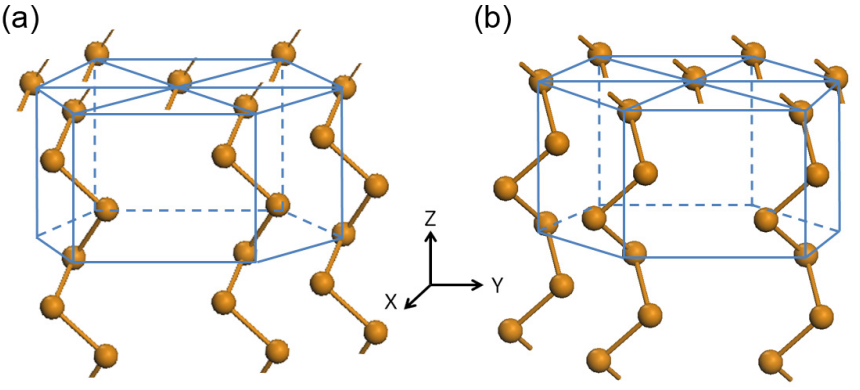

FIG. 1. Crystal structure of bulk tellurium with space groups (a) $P 3_{2} 21$ (no. 154, left-handed) and (b) $P 3_{1} 21$ (no. 152, righthanded). Helical tellurium atomic chains arranged in a hexagonal array spiraling around axes parallel to the $z$ axis. The screw structure of the atoms along the spiral axis for each space group is in the opposite direction.

neighbors. Thus there exists inherent structural anisotropy in the tellurium crystal [49].

\section{COMPUTATIONAL METHOD}

The geometric structure of tellurium crystal is relaxed by using the CASTEP package [50] within the density-functional theory framework. The ultrasoft pseudopotentials are used in the calculations, and the plane-wave cutoff energy is set to be $210 \mathrm{eV}$. The generalized gradient approximation (GGA) of the Perdew-Burke-Ernzerhof (PBE) form [51] is employed for the exchange-correlation functional, and van der Waals interaction correction is considered by using the Tkatchenko and Scheffler method [52].

Time evolution of the wave functions and time-dependent electronic current are studied by using the OCTOPUs package [53], where the Kohn-Sham equations are propagated in real time and real space within the TDDFT framework in the adiabatic local density approximation. We have also tested using a different exchange-correlation functional, i.e., adiabatic GGA (PBE), with pseudopotentials given by Ref. [54], which gives similar results. In all the calculations, the realspace spacing is 0.4 Bohr. The fully relativistic Hartwigsen, Goedecker, and Hutter pseudopotentials are used. A $24 \times$ $24 \times 16$ Monkhorst-Pack $k$-point mesh is used for Brillouinzone sampling.

The laser field is described in the velocity gauge. The vector potential of the circularly polarized laser has the following form:

$$
\mathbf{A}(t)=\frac{\sqrt{I_{0}} c}{\omega} f(t)\left[\frac{\cos (\omega t+\phi)}{\sqrt{1+\epsilon^{2}}} \hat{\mathbf{e}}_{i}+\frac{\epsilon \sin (\omega t+\phi)}{\sqrt{1+\epsilon^{2}}} \hat{\mathbf{e}}_{j}\right],
$$

where $I_{0}=8 \times 10^{11} \mathrm{~W} / \mathrm{cm}^{2}$ is the laser peak intensity in vacuum, $f(t)=\sin ^{2}(\pi t / 2 \tau)$ is the sine-squared pulse envelope with $\tau=20 \mathrm{fs}, \omega$ is the laser photon frequency, $\phi$ is the carrier-envelope phase, $\epsilon$ is the laser ellipticity, $c$ is the light speed in vacuum, and $\hat{\mathbf{e}}_{i}$ and $\hat{\mathbf{e}}_{j}$ with $(i, j) \in$ $\{(x, y),(x, z),(y, z)\}$ are the unit vectors, respectively. We use Ti:sapphire laser pulses with wavelength of $\lambda_{L}=800 \mathrm{~nm}$, corresponding to $\omega=1.55 \mathrm{eV}$. The carrier-envelope phase is taken to be $\phi=0$. Left-handed circularly polarized (LCP) and right-handed circularly polarized (RCP) laser pulses are considered in this work, corresponding to $\epsilon=-1$ and $\epsilon=1$, respectively.

The HHG spectrum is calculated from the time-dependent electronic current $\mathbf{j}(\mathbf{r}, t)$ as

$$
\operatorname{HHG}(\omega)=\left|\mathcal{F} \mathcal{T}\left(\frac{\partial}{\partial t} \int \mathbf{j}(\mathbf{r}, t) d^{3} \mathbf{r}\right)\right|^{2},
$$

where $\mathcal{F} \mathcal{T}$ denotes the Fourier transform.

The total number of excited electrons is calculated by projecting the time-dependent Kohn-Sham states onto the ground-state Kohn-Sham states. As the $n$th state evolves in time, it has some possibility to transit to other states and thus contains other ground-state components. The total number of excited electrons $N_{e x}(t)$ is calculated as

$$
N_{e x}(t)=N_{e}-\int_{\mathrm{BZ}} \sum_{n, m}^{\mathrm{occ}} f_{n, k}\left|\left\langle\phi_{n, k}(t) \mid \phi_{m, k}(0)\right\rangle\right|^{2} d \mathbf{k},
$$

where $N_{e}$ is the total number of electrons in the system, $\phi_{n, k}(t)$ is the time-dependent Kohn-Sham state at the $n$th band at the $k$-point $k, \phi_{m, k}(0)$ is the ground-state $(t=0) \mathrm{Kohn}$-Sham state at the $m$ th band at $k$-point $k, f_{n, k}$ is the occupation of the Kohn-Sham state at the $n$th band at $k$-point $k$, and $\mathrm{BZ}$ denotes integration over the whole Brillouin zone.

\section{CRYSTALLINE SYMMETRIES}

The top view (in the $x-y$ plane) of the crystal structure of bulk tellurium with the space group $P 3_{1} 21$ (right-handed) is shown in Fig. 2(a), while the side views in the $x-z$ and $y-z$ planes are shown in Figs. 2(b)-2(c), respectively. In a series of simulations, we use LCP or RCP laser pulse propagating along the $z, y$, or $x$ directions, i.e., with laser field components in the $x-y, x-z$, or $y-z$ planes. The in-plane HHG spectra are shown in Figs. 2(d)-2(f), corresponding to Figs. 2(a)-2(c), respectively. Different features of appearing harmonic orders are observed, which reflect different crystalline symmetries in each plane. In the $x-y$ plane, the crystal structure exhibits a threefold rotational symmetry [Fig. 2(a)]. Since the circularly polarized laser field can be assumed isotropic in the $x-y$ polarization plane, the laser-crystal interaction system thus possesses a threefold rotational symmetry in this plane. For such a system, the harmonic order $n_{\mathrm{H}}$ obeys a simple selection rule of $n_{\mathrm{H}}=3 m \pm 1(m \in \mathbb{N})$, i.e., every third harmonic is suppressed $[29,55,56]$. The corresponding harmonic spectra shown in Fig. 2(d) agree well with this selection rule. In the $x-z$ plane, the crystal structure lacks an inversion symmetry [Fig. 2(b)]. As a result, both odd and even harmonics are present in the corresponding harmonic spectra [Fig. 2(e)]. In contrast, the projected crystal structure in the $y-z$ plane is centrosymmetric [Fig. 2(c)]. Consequently, only odd harmonics can be observed in the spectra [Fig. 2(f)]. Therefore the feature of allowed harmonic orders of the HHG spectra can be employed to determine the lattice symmetry and crystal orientation of bulk tellurium.

\section{STRUCTURAL CHIRALITY}

Apart from the harmonic orders, another important characteristic of the HHG spectra shown in Figs. 2(d)-2(f) is the 
(a)

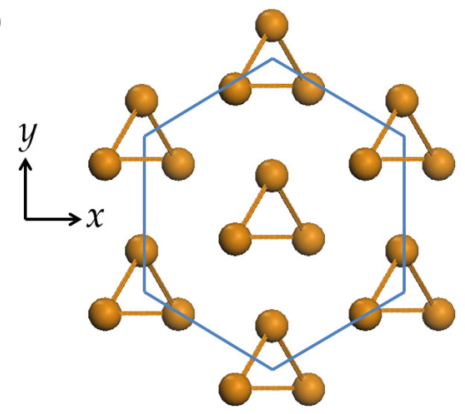

(d)

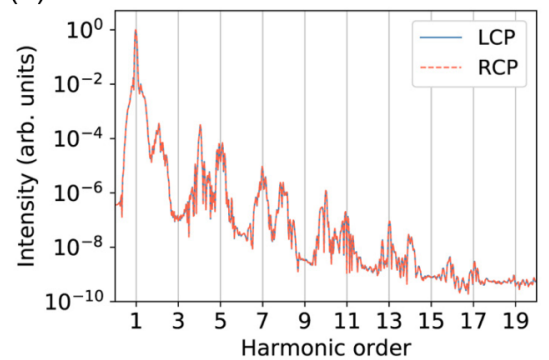

(b)

(c)


(e)

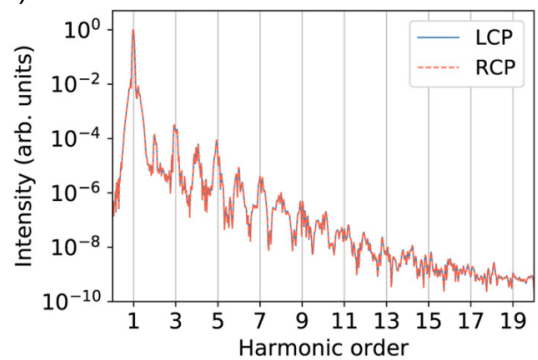

(f)

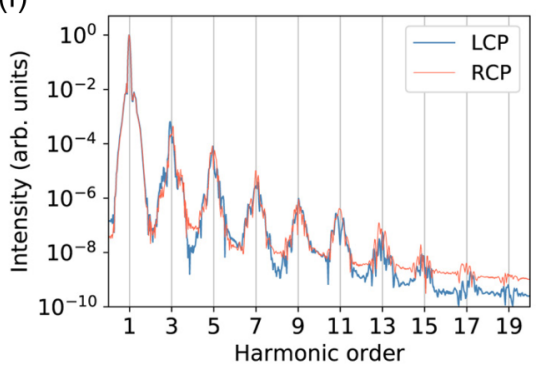

FIG. 2. Orientation-dependent high harmonic generation in the tellurium crystal with the $P 3_{1} 21$ space group. (a-c) Crystal structures in the $x-y$ plane [panel (a); top view], $x-z$ plane [panel (b); side view], and $y-z$ plane [panel (c); side view]. (d-f) High harmonic spectra for different crystal orientations corresponding to $(\mathrm{a}-\mathrm{c})$, respectively. For each crystal orientation, circularly polarized lasers of opposite handedness are incident onto the crystal under normal incidence. LCP and RCP denote the recorded high harmonics driven by left- and right-handed circularly polarized laser pulses, respectively. The presence of harmonic orders reflects the crystalline symmetries in each plane. The laser wavelength is $800 \mathrm{~nm}$ and intensity is $8 \times 10^{11} \mathrm{~W} / \mathrm{cm}^{2}$.

different harmonic-intensity response to the LCP and RCP lasers for different crystal orientations and related lattice structures. While the LCP and RCP laser-driving HHG intensity show no difference in Figs. 2(d) and 2(e), an appreciable $\mathrm{CD}$ effect over a broad range of harmonic orders can be observed in the HHG spectra shown in Fig. 2(f). This CD effect can also be seen clearly from the time evolution of the excited electron numbers and total electronic currents. For the case of an $x-y$ plane, either the number of excited electrons [Fig. 3(a)] or the total currents [Figs. 3(b) and 3(c)] show no difference between LCP and RCP laser pumps. In comparison, for the case of an $y-z$ plane, the dynamics of carrier excitation to the conduction bands during the laser pulse show different responses to LCP and RCP driving lasers in both excitation amplitude and phases [Fig. 3(d)]. This means interacting with such a crystal structure [Fig. 2(c)] lasers of opposite handedness can have different dynamic absorption and ionization probabilities. While the $\mathrm{CD}$ effect on the excitation response is small, a pronounced difference can be seen in the total current [Figs. 3(e) and 3(f)].

The CD effect can be explained by the crystal's structural chirality-induced chiroptical response. Mathematically, a figure is said to have chirality if it cannot be mapped to its mirror image by (proper) rotations and translations alone. As a result, a chiral figure cannot possess an axis of symmetry in two dimensions. Based on this criterion, it is easy to see that the crystal structures in the $x-y$ and $x-z$ planes [Figs. 2(a) and 2(b)] are achiral, while the crystal structure in the $y-z$ plane [Fig. 2(c)] indeed possesses chirality. As circularly polarized lasers are chiral objects exhibiting optical chirality, in their interaction with another chiral object, i.e., the chiral
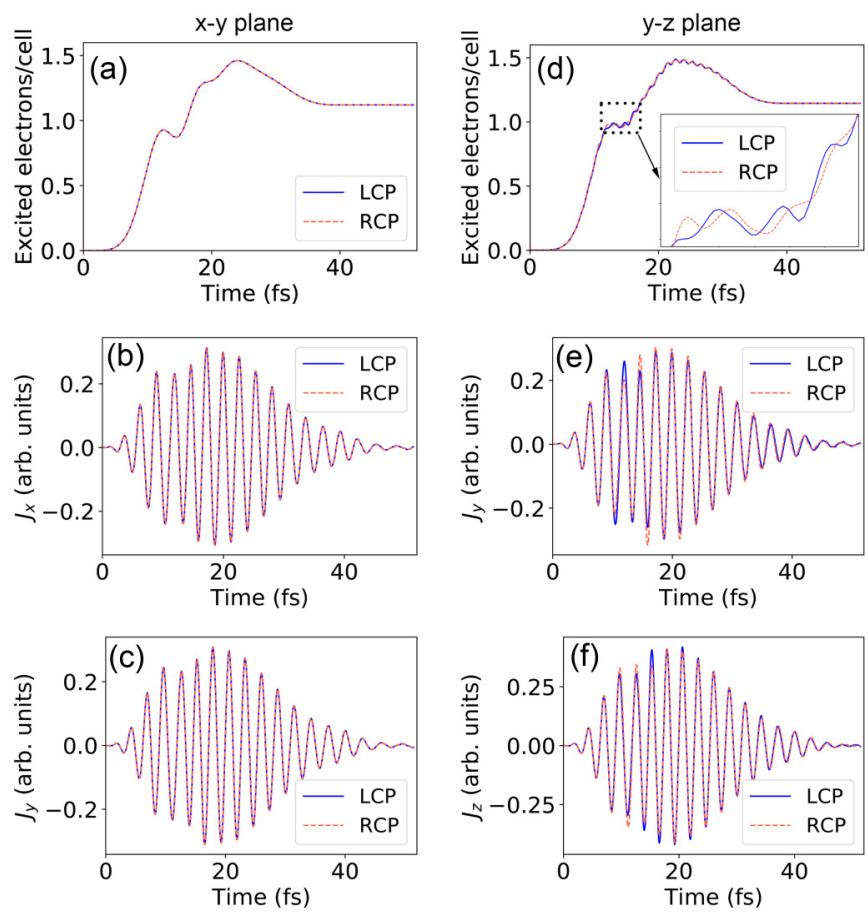

FIG. 3. Circular dichroism effect on the dynamics of electron excitation and electronic current. (a,d) Time evolution of the number of electrons excited to the conduction bands and (b-f) electronic current during the laser pulse. Panels $(\mathrm{a}-\mathrm{c})$ are for laser polarization and crystal structure in the $x-y$ plane, while panels $(\mathrm{d}-\mathrm{f})$ are for the $y-z$ plane. The laser wavelength is $800 \mathrm{~nm}$ and intensity is $8 \times 10^{11}$ $\mathrm{W} / \mathrm{cm}^{2}$. 
crystal, LCP and RCP laser-induced HHG response becomes distinguishable.

In the velocity gauge, the general Hamiltonian can be written as $\hat{H}=\sum_{j}\left[\left(\boldsymbol{p}_{j}+\boldsymbol{A}_{j}\right)^{2} / 2+V_{j}\right]+W$, where $\boldsymbol{p}_{j}=-i \nabla$ is the canonical momentum operator for the $j$ th electron, $\boldsymbol{A}$ is the (chiral) electromagnetic field vector potential, $V$ is the (chiral) Coulomb scalar potential, and $W$ is the interaction between electrons. In this gauge, both electric dipole and nondipole interaction effects are accounted for by the HHG. As atoms are oriented in crystals, here the electric dipole effect is likely to dominate over nondipole effects in the chiral contribution [57,58].

As the applied laser wavelength is much larger than the relevant atomic dimensions, the long-wavelength approximation is adopted in the TDDFT calculations. As such, any spatial dependence of the field is neglected in the simulations, i.e., $\boldsymbol{A}(\boldsymbol{r}, t) \rightarrow \boldsymbol{A}(t)$. Then the field is spatially uniform at any time instant rather than really propagating along the wave vector direction that would require $\boldsymbol{A}(\omega t-\boldsymbol{k} \cdot \boldsymbol{r})$ satisfying the wave equation and depending on both space and time coordinates. This means that the field vector of the circularly polarized light in the simulations, instead of screwing in the three-dimensional (3D) space, rotates only in the polarization planes, which are 2D planes perpendicular to the propagation axis. Consequently, the aforementioned chiroptical response is limited to this two-dimensional (2D) effect. Nevertheless, the above results unambiguously demonstrate that $2 \mathrm{D}$ structural chirality can be probed using circular dichroism of HHG in solids. Therefore the chiral HHG spectroscopy is directly applicable to study atomically thin 2D chiral crystals such as tellurene [59]. Moreover, the results also imply the crystal's 3D structural chirality can be probed by this method. Thus CD signals of HHG in tellurium crystals due to the helical lattice structure along the $z$ axis can be expected in real experiments. This may provide an alternative approach to identify the handedness of elemental crystals such as tellurium or selenium [60].

To further demonstrate the chiroptical response can be attributed to the structural chirality, we compute the CD signals from crystal structures of opposite handedness. Figure 4(a) shows the atomic configurations of tellurium crystal with the $P 3_{1} 21$ (right-handed) and $P 3_{2} 21$ (left-handed) space groups in the $y-z$ plane. Obviously, they are nonsuperimposable mirror images to each other. The quantitative $\mathrm{CD}$ signal is defined as

$$
C D=\frac{I_{R}-I_{L}}{I_{R}+I_{L}}
$$

where $I_{R}$ and $I_{L}$ denote the peak harmonic intensity for each harmonic order driven by the RCP and LCP lasers, respectively. The obtained CD signal for different harmonic orders is shown in Fig. 4(b). The CD asymmetry reaches up to 50\%, much larger than that in molecules $[6,8]$. When the crystal structure is right-handed, more harmonic orders show stronger intensity driven by RCP lasers than by LCP lasers. Switching the handedness of crystal reverses this effect. The CD signal shows a perfect mirroring between the two crystals of opposite handedness, proving that the chiroptical response is indeed due to the structural chirality of the tellurium crystal.

We also plot a set of CD signal data recorded at different driving laser intensities in Fig. 4(c). We see the change of laser (a)

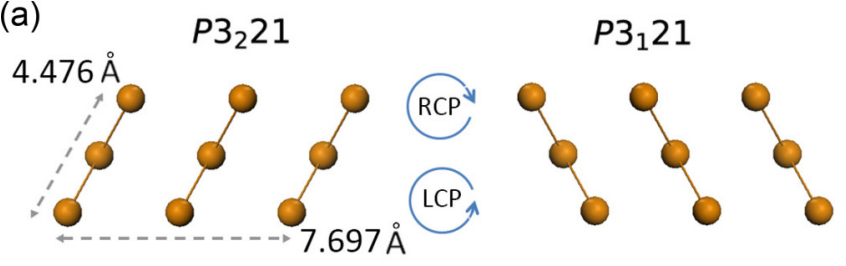

(b)



(c)

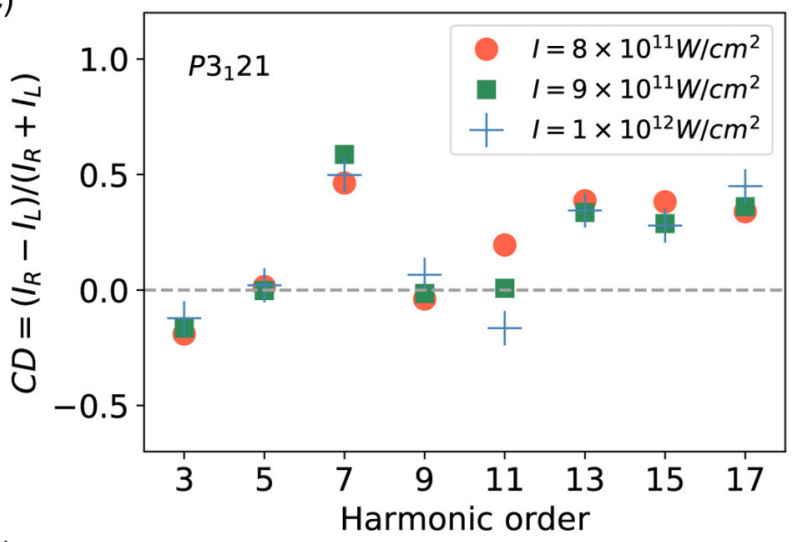

(d)

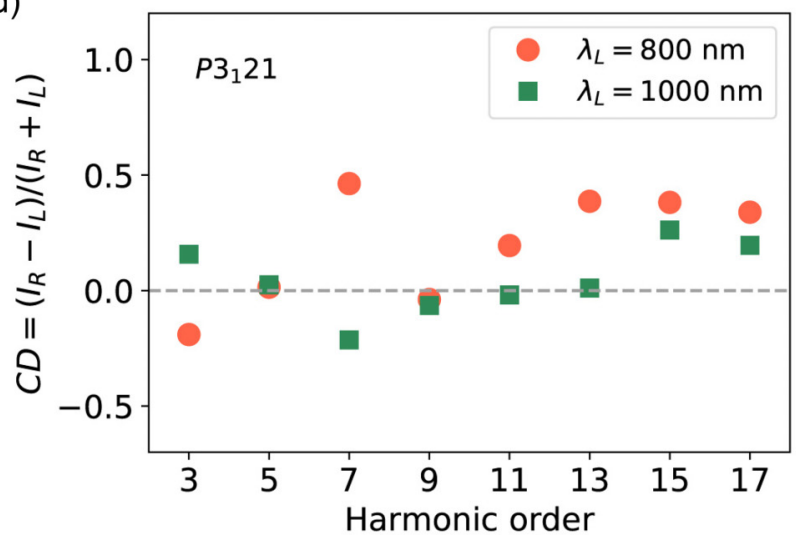

FIG. 4. Chiral response of high harmonic emission. (a) Two enantiomorphic crystal structures of tellurium with space groups $P 3_{1} 21$ (right-handed) and $P 3_{2} 21$ (left-handed) in the $y$ - $z$ plane. Leftand right-handed circularly polarized (LCP and RCP) lasers are also in the same plane. (b) Quantitatively evaluated circular dichroism (CD) signal from the enantiomers for each harmonic order. The laser wavelength is $800 \mathrm{~nm}$ and intensity is $8 \times 10^{11} \mathrm{~W} / \mathrm{cm}^{2}$. CD signal as a function of harmonic photon energy for different (c) laser intensity (with wavelength of $800 \mathrm{~nm}$ ) and (d) laser wavelength (with intensity of $8 \times 10^{11} \mathrm{~W} / \mathrm{cm}^{2}$ ). 

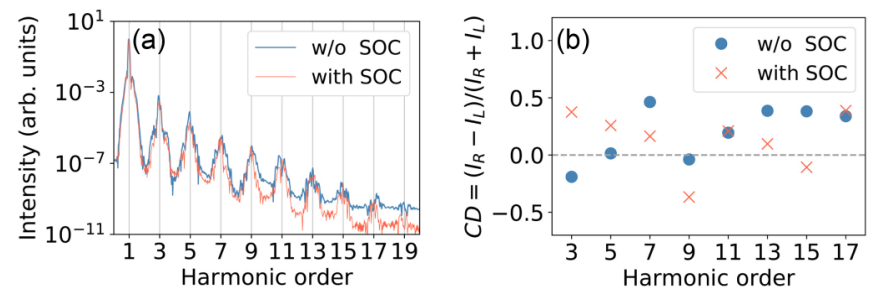

FIG. 5. Spin-orbit coupling (SOC) effect on HHG. (a) High harmonic spectra and (b) CD signals obtained with (red) and without (blue) considering SOC effect. The other simulation parameters are the same with those in Fig. 2(f).

intensity only has a small impact on the CD signals, in contrast to previous studies on chiral HHG from gas molecules where CD signals show substantial changes with a minor change of laser intensity [8]. This result suggests in this parameter regime chiral electronic dynamics taking place during the HHG process having minor contribution to the observed chiral signals. Instead, effects originating from the static structure of the crystals dominate the chiroptical response. In comparison, the dependence of $\mathrm{CD}$ signal on laser wavelength is relatively more sensitive, as shown in Fig. 4(d), implying an increased contribution from electronic dynamics. Thus both static structure and dynamic effects can contribute to the $\mathrm{CD}$ signal.

Finally, we address the effect of spin-orbit coupling (SOC). Previous studies suggest that the SOC effect could play an important role in the electronic properties of tellurium and trigger exotic electromagnetic effects [61,62]. We have carried out additional simulations with the SOC effect included and other parameters the same as those in Fig. 2(f). Figure 5(a) shows the peak harmonic intensity is lowered as compared to that without SOC, while the presence of harmonic orders remains the same, since it is determined by the lattice symmetry. Figure 5(b) shows a significant CD signal is still induced when the SOC effect is considered, albeit maybe with a different value for each harmonic order. As in the present work we focus on lattice structural chirality, the SOC effect would not change our main conclusion qualitatively that structural chirality of crystals can lead to the CD effect in HHG from the material. Studying the influence of electronic and spin structures in tellurium on HHG would be an interesting future work.

\section{CONCLUSIONS}

In conclusion, we demonstrate through ab initio TDDFT calculations that chiral HHG can be induced from interaction of circularly polarized lasers with chiral crystals. While the allowed harmonic orders reflect the lattice symmetries, CD signals of harmonic intensity shed light on the structural chirality. It is worth noting that there exists different phases for $2 \mathrm{D}$ tellurene (atomically thin tellurium), e.g., the $\alpha, \beta, \gamma$, and $\delta$ phase [63]. As the different phases of tellurene exhibit different symmetries and chirality, the circularly polarized HHG spectroscopy is also potentially useful for characterizing the phases and phase transition dynamics of this emerging $2 \mathrm{D}$ material.

\section{ACKNOWLEDGMENTS}

This work was supported in part by the National Natural Science Foundation of China (Grant No. 11705185), the Presidential Fund of the China Academy of Engineering Physics (Grant No. YZJJLX2017002), and the Fundamental Research Funds for the Central Universities.
[1] S. Riva, Chirality in metals: An asymmetrical journey among advanced functional materials, Mater. Sci. Technol. 33, 795 (2017).

[2] Circular Dichroism and the Conformational Analysis of Biomolecules, edited by G. D. Fasman (Springer, New York, 2013).

[3] N. Böwering, T. Lischke, B. Schmidtke, N. Müller, T. Khalil, and U. Heinzmann, Asymmetry in Photoelectron Emission from Chiral Molecules Induced by Circularly Polarized Light, Phys. Rev. Lett. 86, 1187 (2001).

[4] C. Lux, M. Wollenhaupt, T. Bolze, Q. Liang, J. Köhler, C. Sarpe, and T. Baumert, Circular dichroism in the photoelectron angular distributions of camphor and fenchone from multiphoton ionization with femtosecond laser pulses, Angew. Chem., Int. Ed. 51, 5001 (2012).

[5] P. Fischer and F. Hache, Nonlinear optical spectroscopy of chiral molecules, Chirality 17, 421 (2005).

[6] R. Cireasa, A. E. Boguslavskiy, B. Pons, M. C. H. Wong, D. Descamps, S. Petit, H. Ruf, N. Thiré, A. Ferré, J. Suarez, J. Higuet, B. E. Schmidt, A. F. Alharbi, F. Légaré, V. Blanchet, B. Fabre, S. Patchkovskii, O. Smirnova, Y. Mairesse, and V. R. Bhardwaj, Probing molecular chirality on a subfemtosecond timescale, Nat. Phys. 11, 654 (2015).
[7] O. Smirnova, Y. Mairesse, and S. Patchkovskii, Opportunities for chiral discrimination using high harmonic generation in tailored laser fields, J. Phys. B 48, 234005 (2015).

[8] D. Baykusheva and H. J. Wörner, Chiral Discrimination through Bielliptical High-Harmonic Spectroscopy, Phys. Rev. X 8, 031060 (2018).

[9] O. Neufeld and O. Cohen, Optical Chirality in Nonlinear Optics: Application to High Harmonic Generation, Phys. Rev. Lett. 120, 133206 (2018).

[10] Y. Harada, E. Haraguchi, K. Kaneshima, and T. Sekikawa, Circular dichroism in high-order harmonic generation from chiral molecules, Phys. Rev. A 98, 021401(R) (2018).

[11] O. Neufeld, D. Ayuso, P. Decleva, M. Y. Ivanov, O. Smirnova, and O. Cohen, Ultrasensitive Chiral Spectroscopy by Dynamical Symmetry Breaking in High Harmonic Generation, Phys. Rev. X 9, 031002 (2019).

[12] H. D. Flack, Chiral and achiral crystal structure, Helv. Chim. Acta 86, 905 (2003).

[13] N. A. Spaldin and M. Fiebig, The renaissance of magnetoelectric multiferroics, Science 309, 391 (2005).

[14] S. Mühlbauer, B. Binz, F. Jonietz, C. Pfleiderer, A. Rosch, A. Neubauer, R. Georgii, and P. Böni, Skyrmion lattice in a chiral magnet, Science 323, 915 (2009). 
[15] T. Yoda, T. Yokoyama, and S. Murakami, Current-induced orbital and spin magnetizations in crystals with helical structure, Sci. Rep. 5, 12024 (2015).

[16] S. S. Tsirkin, P. A. Puente, and I. Souza, Gyrotropic effects in trigonal tellurium studied from first principles, Phys. Rev. B 97, 035158 (2018).

[17] G. Chang, B. J. Wieder, F. Schindler, D. S. Sanchez, I. Belopolski, S.-M. Huang, B. Singh, D. Wu, T.-R. Chang, T. Neupert, S.-Y. Xu, H. Lin, and M. Z. Hasan, Topological quantum properties of chiral crystals, Nat. Mater. 17, 978 (2018).

[18] D. S. Sanchez, I. Belopolski, T. A. Cochran, X. Xu, J.-X. Yin, G. Chang, W. Xie, K. Manna, V. Süß, C.-Y. Huang, N. Alidoust, D. Multer, S. S. Zhang, N. Shumiya, X. Wang, G.-Q. Wang, T.-R. Chang, C. Felser, S.-Y. Xu, S. Jia, H. Lin, and M. Z. Hasan, Topological chiral crystals with helicoid-arc quantum states, Nature (London) 567, 500 (2019).

[19] S. Ghimire, A. D. DiChiara, E. Sistrunk, P. Agostini, L. F. DiMauro, and D. A. Reis, Observation of high-order harmonic generation in a bulk crystal, Nat. Phys. 7, 138 (2011).

[20] S. Ghimire and D. A. Reis, High-harmonic generation from solids, Nat. Phys. 15, 10 (2019).

[21] N. Tancogne-Dejean, O. D. Mücke, F. X. Kärtner, and A. Rubio, Ellipticity dependence of high-harmonic generation in solids originating from coupled intraband and interband dynamics, Nat. Commun. 8, 745 (2017).

[22] F. Langer, M. Hohenleutner, U. Huttner, S. W. Koch, M. Kira, and R. Huber, Symmetry-controlled temporal structure of highharmonic carrier fields from a bulk crystal, Nat. Photonics 11, 227 (2017).

[23] T. J. Hammond, S. Monchocë, C. Zhang, G. Vampa, D. Klug, A. Y. Naumov, D. M. Villeneuve, and P. B. Corkum, Integrating solids and gases for attosecond pulse generation, Nat. Photonics 11, 594 (2017).

[24] M. Sivis, M. Taucer, G. Vampa, K. Johnston, A. Staudte, A. Y. Naumov, D. M. Villeneuve, C. Ropers, and P. B. Corkum, Tailored semiconductors for high-harmonic optoelectronics, Science 357, 303 (2017).

[25] M. Garg, H. Y. Kim, and E. Goulielmakis, Ultimate waveform reproducibility of extreme-ultraviolet pulses by high-harmonic generation in quartz, Nat. Photonics 12, 291 (2018).

[26] N. Yoshikawa, T. Tamaya, and K. Tanaka, High-harmonic generation in graphene enhanced by elliptically polarized light excitation, Science 356, 736 (2017).

[27] M. Taucer, T. J. Hammond, P. B. Corkum, G. Vampa, C. Couture, N. Thiré, B. E. Schmidt, F. Légaré, H. Selvi, N. Unsuree, B. Hamilton, T. J. Echtermeyer, and M. A. Denecke, Nonperturbative harmonic generation in graphene from intense midinfrared pulsed light, Phys. Rev. B 96, 195420 (2017).

[28] M. Baudisch, A. Marini, J. D. Cox, T. Zhu, F. Silva, S. Teichmann, M. Massicotte, F. Koppens, L. S. Levitov, F. J. García de Abajo, and B. Jens, Ultrafast nonlinear optical response of dirac fermions in graphene, Nat. Commun. 9, 1018 (2018).

[29] Z.-Y. Chen and R. Qin, Circularly polarized extreme ultraviolet high harmonic generation in graphene, Opt. Express 27, 3761 (2019).

[30] R. Qin and Z.-Y. Chen, Strain-controlled high harmonic generation with Dirac fermions in silicene, Nanoscale 10, 22593 (2018).
[31] Z.-Y. Chen and R. Qin, Strong-field nonlinear optical properties of monolayer black phosphorus, Nanoscale 11, 16377 (2019).

[32] M.-X. Guan, C. Lian, S.-Q. Hu, H. Liu, S.-J. Zhang, J. Zhang, and S. Meng, Cooperative evolution of intraband and interband excitations for high-harmonic generation in strained $\mathrm{MoS}_{2}$, Phys. Rev. B 99, 184306 (2019).

[33] M. Guan, S. Hu, H. Zhao, C. Lian, and S. Meng, Toward attosecond control of electron dynamics in two-dimensional materials, Appl. Phys. Lett. 116, 043101 (2020).

[34] N. Yoshikawa, K. Nagai, K. Uchida, Y. Takaguchi, S. Sasaki, Y. Miyata, and K. Tanaka, Interband resonant high-harmonic generation by valley polarized electron-hole pairs, Nat. Commun. 10, 3709 (2019).

[35] N. Tancogne-Dejean and A. Rubio, Atomic-like high-harmonic generation from two-dimensional materials, Sci. Adv. 4, eaao5207 (2018).

[36] G. Le Breton, A. Rubio, and N. Tancogne-Dejean, Highharmonic generation from few-layer hexagonal boron nitride: Evolution from monolayer to bulk response, Phys. Rev. B 98, 165308 (2018).

[37] G. Vampa, C. R. McDonald, G. Orlando, D. D. Klug, P. B. Corkum, and T. Brabec, Theoretical Analysis of HighHarmonic Generation in Solids, Phys. Rev. Lett. 113, 073901 (2014).

[38] T. T. Luu, M. Garg, S. Y. Kruchinin, A. Moulet, M. T. Hassan, and E. Goulielmakis, Extreme ultraviolet high-harmonic spectroscopy of solids, Nature (London) 521, 498 (2015).

[39] N. Tancogne-Dejean, O. D. Mücke, F. X. Kärtner, and A. Rubio, Impact of the Electronic Band Structure in High-Harmonic Generation Spectra of Solids, Phys. Rev. Lett. 118, 087403 (2017).

[40] N. Klemke, N. Tancogne-Dejean, G. M. Rossi, Y. Yang, F. Scheiba, R. E. Mainz, G. Di Sciacca, A. Rubio, F. X. Kärtner, and O. D. Mücke, Polarization-state-resolved high-harmonic spectroscopy of solids, Nat. Commun. 10, 1319 (2019).

[41] Y. S. You, D. A. Reis, and S. Ghimire, Anisotropic highharmonic generation in bulk crystals, Nat. Phys. 13, 345 (2017).

[42] G. Vampa, T. J. Hammond, N. Thiré, B. E. Schmidt, F. Légaré, C. R. McDonald, T. Brabec, D. D. Klug, and P. B. Corkum, AllOptical Reconstruction of Crystal Band Structure, Phys. Rev. Lett. 115, 193603 (2015).

[43] A. A. Lanin, E. A. Stepanov, A. B. Fedotov, and A. M. Zheltikov, Mapping the electron band structure by intraband high-harmonic generation in solids, Optica 4, 516 (2017).

[44] T. T. Luu and H. J. Wörner, Measurement of the Berry curvature of solids using high-harmonic spectroscopy, Nat. Commun. 9, 916 (2018).

[45] R. E. F. Silva, I. V. Blinov, A. N. Rubtsov, O. Smirnova, and M. Ivanov, High-harmonic spectroscopy of ultrafast many-body dynamics in strongly correlated systems, Nat. Photonics 12, 266 (2018).

[46] D. Bauer and K. K. Hansen, High-Harmonic Generation in Solids With and Without Topological Edge States, Phys. Rev. Lett. 120, 177401 (2018).

[47] R. E. F. Silva, A. Jiménez-Galán, B. Amorim, O. Smirnova, and M. Ivanov, Topological strong-field physics on sub-laser-cycle timescale, Nat. Photonics 13, 849 (2019).

[48] R. H. Asendorf, Space group of tellurium and selenium, J. Chem. Phys. 27, 11 (1957). 
[49] H. Peng, N. Kioussis, and D. A. Stewart, Anisotropic lattice thermal conductivity in chiral tellurium from first principles, Appl. Phys. Lett. 107, 251904 (2015).

[50] S. J. Clark, M. D. Segall, C. J. Pickard, P. J. Hasnip, M. J. Probert, K. Refson, and M. C. Payne, First principles methods using Castep, Z. Kristallographie 220, 567 (2005).

[51] J. P. Perdew, K. Burke, and M. Ernzerhof, Generalized Gradient Approximation Made Simple, Phys. Rev. Lett. 77, 3865 (1996).

[52] A. Tkatchenko and M. Scheffler, Accurate Molecular Van der Waals Interactions from Ground-State Electron Density and Free-Atom Reference Data, Phys. Rev. Lett. 102, 073005 (2009).

[53] X. Andrade, D. A. Strubbe, U. D. Giovannini, A. H. Larsen, M. J. T. Oliveira, J. Alberdi-Rodriguez, A. Varas, I. Theophilou, N. Helbig, M. Verstraete, L. Stella, F. Nogueira, A. AspuruGuzik, A. Castro, M. A. L. Marques, and A. Rubio, Real-space grids and the Octopus code as tools for the development of new simulation approaches for electronic systems, Phys. Chem. Chem. Phys. 17, 31371 (2015).

[54] M. Schlipf and F. Gygi, Optimization algorithm for the generation of ONCV pseudopotentials, Comput. Phys. Commun. 196, 36 (2015).

[55] N. Saito, P. Xia, F. Lu, T. Kanai, J. Itatani, and N. Ishii, Observation of selection rules for circularly polarized fields in high-harmonic generation from a crystalline solid, Optica 4, 1333 (2017).

[56] Z.-Y. Chen, Spectral control of high harmonics from relativistic plasmas using bicircular fields, Phys. Rev. E 97, 043202 (2018).
[57] Y. Mairesse, N. Dudovich, J. Levesque, M. Y. Ivanov, P. B. Corkum, and D. M. Villeneuve, Electron wavepacket control with elliptically polarized laser light in high harmonic generation from aligned molecules, New J. Phys. 10, 025015 (2008).

[58] D. Wang, X. Zhu, X. Liu, L. Li, X. Zhang, P. Lan, and P. Lu, High harmonic generation from axial chiral molecules, Opt. Express 25, 23502 (2017).

[59] W. Wu, G. Qiu, Y. Wang, R. Wang, and P. Ye, Tellurene: Its physical properties, scalable nanomanufacturing, and device applications, Chem. Soc. Rev. 47, 7203 (2018).

[60] Y. Tanaka, S. P. Collins, S. W. Lovesey, M. Matsumami, T. Moriwaki, and S. Shin, Determination of the absolute chirality of tellurium using resonant diffraction with circularly polarized X-rays, J. Phys.: Condens. Matter 22, 122201 (2010).

[61] M. Sakano, M. Hirayama, T. Takahashi, S. Akebi, M. Nakayama, K. Kuroda, K. Taguchi, T. Yoshikawa, K. Miyamoto, T. Okuda, K. Ono, H. Kumigashira, T. Ideue, Y. Iwasa, N. Mitsuishi, K. Ishizaka, S. Shin, T. Miyake, S. Murakami, T. Sasagawa, and T. Kondo, Radial Spin Texture in Elemental Tellurium with Chiral Crystal Structure, Phys. Rev. Lett. 124, 136404 (2020).

[62] D. Rodriguez, A. A. Tsirlin, T. Biesner, T. Ueno, T. Takahashi, K. Kobayashi, M. Dressel, and E. Uykur, Two Linear Regimes in Optical Conductivity of a Type-I Weyl Semimetal: The Case of Elemental Tellurium, Phys. Rev. Lett. 124, 136402 (2020).

[63] Y. Xiang, S. Gao, R.-G. Xua, W. Wu, and Y. Leng, Phase transition in two-dimensional tellurene under mechanical strain modulation, Nano Energy 58, 202 (2019). 